\title{
Off-label use of duct occluder in transcatheter closure of secundum atrial septal defect with no rim to right pulmonary vein
}

\author{
Chaowu Yan, PhD, MD, ${ }^{a}$ Shiguo Li, PhD, MD, ${ }^{a}$ Huijun Song, PhD, MD,${ }^{a}$ Jinglin Jin, PhD, MD,
} Hong Zheng, $\mathrm{PhD}, \mathrm{MD},{ }^{a}$ Cheng Wang, $\mathrm{PhD}, \mathrm{MD},{ }^{\mathrm{b}}$ and Shihua Zhao, $\mathrm{PhD}, \mathrm{MD}^{\mathrm{c}}$

\section{ABSTRACT}

Objective: Transcatheter closure is usually contraindicated in secundum atrial septal defect with no rim to right pulmonary vein. The morphology of an atrial septal defect is special in these patients, and the off-label use of a duct occluder might make transcatheter closure possible with the assistance of a personalized heart model. However, the related data are absent. This study was performed to investigate the off-label use of a duct occluder in the transcatheter closure of an atrial septal defect with no right pulmonary vein rim.

Methods: Seven consecutive patients with an atrial septal defect ( 2 male/5 female; $40.1 \pm 15.2$ years) with no right pulmonary vein rim were referred for attempted transcatheter closure, and a personalized heart model was produced on the basis of multislice computed tomography images. With the elastic heart model, in vitro trial occlusion was performed with a duct occluder for preoperative evaluation, and the results were compared with postoperative outcomes.

Results: A single atrial septal defect was identified in 6 patients, and a double atrial septal defect was identified in another patient (1 atrial septal defect with no right pulmonary vein rim and 1 with sufficient surrounding rims). The size of the atrial septal defect was $13.4 \pm 2.2 \mathrm{~mm}$, and the in vitro balloon-stretched diameter was $20.2 \pm 3.2 \mathrm{~mm}$ in the heart model. In vitro trial occlusion was achieved in all patients, and subsequent in vivo transcatheter closure was performed successfully with the duct occluder of the same size $(24.3 \pm 3.1 \mathrm{~mm})$. During follow-up $(1.57 \pm 0.84$ years $)$, mild pericardial effusion was identified in 2 patients and disappeared within 3 months. No other complications occurred.

Conclusions: By using a personalized heart model, the off-label use of a duct occluder is feasible and safe in the transcatheter closure of an atrial septal defect with no right pulmonary vein rim. Further research is required to evaluate the long-term outcomes. (J Thorac Cardiovasc Surg 2019;157:1603-8)

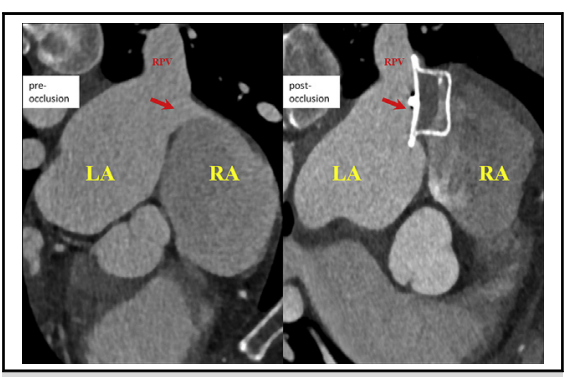

Duct occluder in transcatheter closure of ASD with no RPV rim.

Central Message

Assisted with personalized heart model, the offlabel use of the duct occluder is feasible and safe in transcatheter closure of ASD with no RPV rim.

\section{Perspective}

In ASD with no RPV rim, transcatheter closure is feasible and safe with the off-label use of the duct occluder. On the basis of the personalized heart model, in vitro measurement and trial occlusion provided an excellent method for preoperative evaluation. Further research is needed to investigate the long-term outcome.

\footnotetext{
From the a Department of Structural Heart Disease, Cardiovascular Institute and Fuwai Hospital, National Center for Cardiovascular Diseases, Chinese Academy of Medical Sciences and Peking Union Medical College, Beijing, China; ${ }^{\mathrm{b}}$ Department of Cardiology, Affiliated Hospital of Xuzhou Medical University, Quanshan District, Xuzhou City, China; and ${ }^{\mathrm{c}}$ Radiology Imaging Center, Cardiovascular Institute and Fuwai Hospital, National Center for Cardiovascular Diseases, Chinese Academy of Medical Sciences and Peking Union Medical College, Beijing, China.

This work was supported by Beijing Municipal Science and Technology Commission (Z171100001017194), National Natural Science Foundation of China (81670283), and Beijing Natural Science Foundation (7162160).

Received for publication Sept 16, 2018; revisions received Oct 28, 2018; accepted for publication Nov 12, 2018; available ahead of print Feb 1, 2019.

Address for reprints: Chaowu Yan, $\mathrm{PhD}$, MD, and Shihua Zhao, PhD, MD, Department of Structural Heart Disease, Fuwai Hospital, 167 Beilishi Rd, Beijing 100037 China (E-mail: chaowuyan@163.com or cjr.zhaoshihua@vip.163.com). $0022-5223 / \$ 36.00$

Copyright (c) 2018 by The American Association for Thoracic Surgery

https://doi.org/10.1016/j.jtcvs.2018.11.093
}

In secundum atrial septal defect (ASD) with no rim to the right pulmonary vein (RPV), transcatheter closure is usually contraindicated with an atrial septal occluder. ${ }^{1-7}$ Lack of a posterior rim to the RPV makes the implantation of

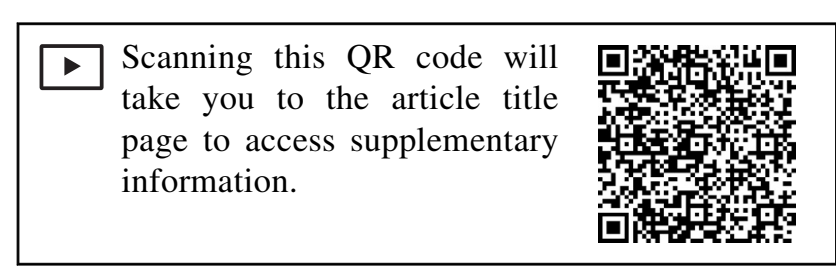




\section{Abbreviations and Acronyms \\ ASD = atrial septal defect \\ MSCT $=$ multislice computed tomography \\ $\mathrm{RPV}=$ right pulmonary vein \\ 3D $=3$-dimensional \\ TTE $=$ transthoracic echocardiography}

the atrial septal occluder almost impossible. Although there is no rim to the RPV, the RPV orifice might act as an effective support point, which probably allows the retention disc of the duct occluder to engage and fix. In view of the potential risks (device embolization, obstruction of RPV, and tissue erosion), up to now, the related attempt is still absent in these patients.

Our previous study proved that in vitro trial occlusion, based on a personalized heart model, provided a feasible method to predict the successful transcatheter closure of complex ASDs. ${ }^{8}$ With the assistance of a personalized heart model, we hypothesized that the duct occluder would have the potential to close the ASD with no RPV rim. In this study, the off-label use of a duct occluder was investigated in these patients.

\section{MATERIALS AND METHODS \\ Study Patients}

From March 2015 to March 2018, 7 consecutive patients with ASDs ( 2 male/5 female; age $40.1 \pm 15.2$ years) with no rim to RPV (right inferior pulmonary vein) were referred for attempted transcatheter closure. All patients underwent a routine clinical examination, and each patient had been identified by Doppler echocardiography to have an ASD with no RPV rim, together with right atrial and right ventricular volume overload. Patients $(n=4)$ with an in vitro balloon-stretched diameter of ASD greater than $25 \mathrm{~mm}(\mathrm{n}=1)$, other coexisting cardiovascular malformations $(n=2)$, and inadequate image quality $(n=1)$ were excluded from the study. Multislice computed tomography (MSCT) was performed to evaluate the surrounding rims of the ASD, and a personalized heart model was produced on the basis of the MSCT end-systolic images. The in vitro measurement and in vitro trial occlusion were carried out in the elastic rubber model for preoperative evaluation, and the results were compared with postoperative outcomes. The study was approved by our hospital research ethics committee, and informed consent was obtained from each patient.

\section{Multislice Computed Tomography Examination and 3-Dimensional Printing}

Electrocardiographic-gated contrast-enhanced MSCT (SOMATOM Definition Flash, Siemens Healthcare, Erlangen, Germany) was performed to assess the ASDs in all patients (slice thickness $0.75 \mathrm{~mm}$ ). ${ }^{8,9}$ After the examination, 10 phases were reconstructed through the cardiac cycle, with the RR interval divided into $10 \%$ increments. The 3-dimensional (3D) printing was based on the MSCT end-systolic images. The overall process of 3D printing included 3D image reconstruction, digital preparation (Video 1), 3D printing, and postprinting finishing work. Digital preparation was accomplished with Mimics 17.0 software (Materialise, Leuven,

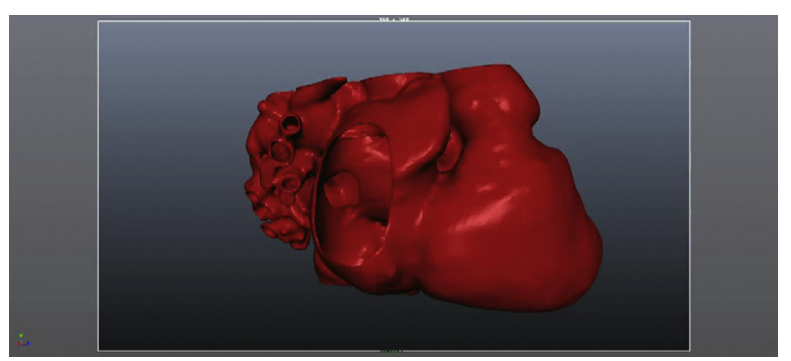

VIDEO 1. The 3D digital model showed the ASD with no rim to the RPV. The other surrounding rims were sufficient. Video available at: https://www.jtcvs.org/article/S0022-5223(18)33239-2/fulltext.

Belgium) to evaluate the anatomic structure of ASD. The final STL files generated via digital preparation were imported into an Objet350 Connex3 (STRATASYS, Eden Prairie, Minn) 3D printer. The personalized heart model was made of elastic rubber, which was the mixture of 3 kinds of photosensitive resin materials: TangoPlus FLX930, VeroFlex Yellow RGD893, and VeroMagenta RGD851 (STRATASYS). With Objet Studio software (STRATASYS, Eden Prairie, Minn), 30 shore hardness was set in the model, and the blending ratio of 3 materials was calculated automatically. Finally, the elastic heart model allowed the detailed observation, measurement with the sizing balloon, and in vitro trial occlusion.

\section{In Vitro Measurement and Trial Occlusion}

On the basis of the elastic heart model, the balloon-stretched diameter of the ASD was determined under fluoroscopy (Amplatzer sizing balloon II, St Jude Medical, St Paul, Minn). With the balloon inflated (contrast agent diluted with saline at a ratio of 1/3), the angulation of the X-ray tube was changed to align the balloon in its longest diameter and to align the "waist" on the balloon. According to the "waist" (balloon-stretched diameter) formed on the sizing balloon, trial occlusion was performed with the occluder $4 \mathrm{~mm}$ larger than the stretched diameter (occluder diameter was defined as the narrowest segment of occluder, namely, the pulmonary segment of duct occluder). Cera duct occluders (Lifetech Scientific Co,

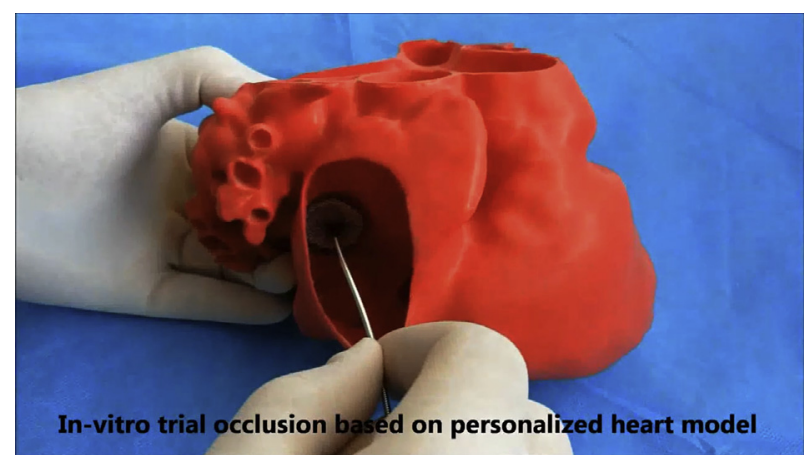

VIDEO 2. The associated operative procedure. The personalized heart model was created for preoperative evaluation. On the basis of the elastic heart model, in vitro measurement and trial occlusion were performed to determine the appropriate device. Then, successful transcatheter closure was performed with a device of the same size in the patient. After the deployment, the duct occluder and adjacent structures were evaluated with repeat TTE and pulmonary arteriography (in the levophase). Video available at: https://www.jtcvs.org/article/S0022-5223(18)33239-2/fulltext. 
TABLE 1. Clinical characteristics of patients with atrial septal defect with no right pulmonary vein $\operatorname{rim}(\mathbf{n}=7)$

\begin{tabular}{|c|c|c|c|c|c|c|c|c|}
\hline Patient no. (n) & Age (y) & Weight (kg) & $\begin{array}{c}\text { ASD size } \\
(\mathbf{m m}, \text { TTE) }\end{array}$ & $\begin{array}{l}\text { In vitro balloon- } \\
\text { stretched } \\
\text { diameter (mm) }\end{array}$ & $\begin{array}{l}\text { Size of selected } \\
\text { occluder (mm) }\end{array}$ & $\begin{array}{c}\text { Postoperative } \\
\text { diameter of } \\
\text { occluder }(\mathbf{m m})\end{array}$ & Qp/Qs & Follow-up (y) \\
\hline $1 *$ & 45 & 74 & 15 & 21.8 & 26 & 22 & 1.8 & 2 \\
\hline $2^{*}$ & 48 & 57 & $13,9 \dagger$ & 22.3 & 26 & 23 & 2.4 & 2 \\
\hline 3 & 8 & 35 & 9.8 & 15.7 & 20 & 16.1 & 2.2 & 3 \\
\hline 4 & 52 & 43 & 15.6 & 24.5 & 28 & 26.3 & 2.6 & 1.5 \\
\hline 5 & 51 & 64 & 12 & 16.5 & 20 & 17.4 & 1.7 & 1 \\
\hline 6 & 39 & 60 & 12.4 & 20 & 24 & 22 & 1.9 & 1 \\
\hline 7 & 38 & 55 & 15.7 & 20.6 & 26 & 22.8 & 1.6 & 0.5 \\
\hline Total $(n)=7$ & $40.1[15.2]$ & $55.4[13.0]$ & 13.4 [2.2] & $20.2[3.2]$ & $24.3[3.1]$ & $21.4[3.5]$ & $2.03[0.38]$ & $1.57[0.84]$ \\
\hline
\end{tabular}

Values are given as mean [standard deviation]. $A S D$, Atrial septal defect; TTE, transthoracic echocardiography; $Q p / Q s$, ratio of pulmonary blood flow to systemic blood flow. *Mild pericardial effusion was detected after transcatheter closure of ASD. $†$ The defect had sufficient rims and the following measurement referred to the ASD with no RPV $\operatorname{rim}(13 \mathrm{~mm})$

Ltd, Shenzhen, China; available size. 4-32 mm) were used in this study, and the device stability was evaluated with the push-pull test.

\section{Right Heart Catheterization and In Vivo Transcatheter Closure}

After percutaneous puncture of the femoral vein (6F Introducer, Cordis, Miami, Fla) under local anesthesia, all patients underwent routine right heart catheterization with a 6F MPA2 catheter (Cordis). Complete hemodynamic data and blood samples were obtained, and the measurements included mean right atrial pressure, right ventricular pressure, systolic pulmonary arterial pressure, diastolic pulmonary arterial pressure, and mean pulmonary arterial pressure. The catheter was also introduced into the

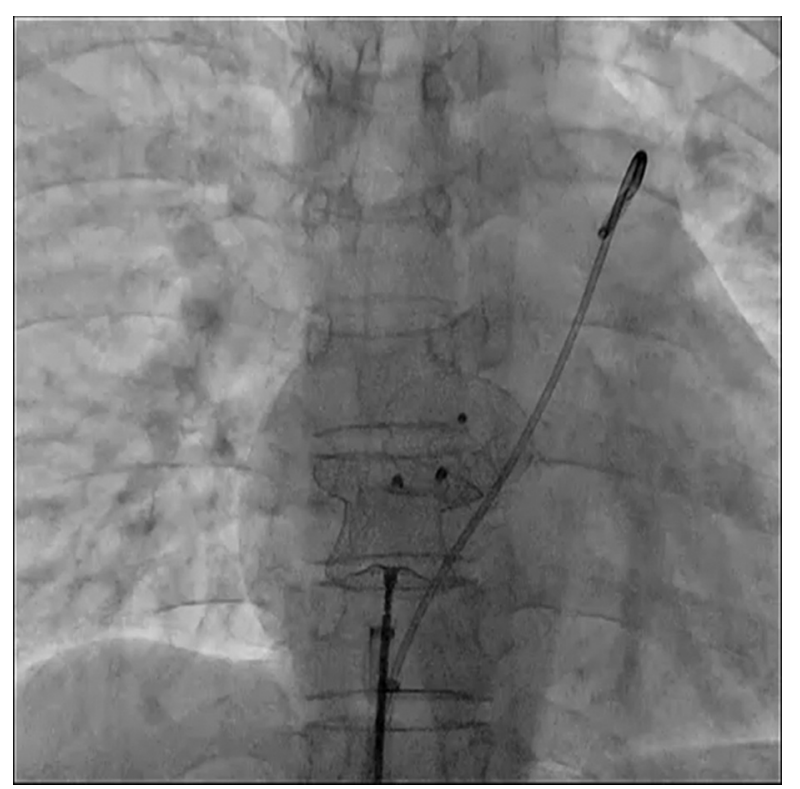

VIDEO 3. In the patient with double ASDs, the first ASD with sufficient rims was closed using a 16-mm atrial septal occluder, and the second ASD with no rim to the RPV was closed successfully using a 26/28 Cera (Lifetech Scientific Co, Ltd, Shenzhen, China) duct occluder. The levophase of pulmonary arteriography excluded the residual shunt and obstruction of the RPV. Video available at: https://www.jtcvs.org/article/ S0022-5223(18)33239-2/fulltext. left atrium through the ASD to obtain the mean left atrial pressure. According to the oxymetric principle of Fick, the pulmonary blood flow, systemic blood flow, ratio of pulmonary to systemic blood flow, and pulmonary vascular resistance were calculated. Occluder selection was based on the in vitro trial occlusion (using the duct occluder of the same size). Through the delivery sheath (into the left superior pulmonary vein), the device was advanced to the left atrium. Then, the sheath was gently withdrawn to deploy the retention disk only, after which the cable and delivery sheath were pulled as 1 unit under the guidance of fluoroscopy until the retention disk was against the left side of the septum. After the deployment, an obvious "waist" must be formed in the occluder, and the push-pull test was performed to test device stability (Video 2). The postoperative diameter of the occluder (the narrowest waist) was measured with fluoroscopy, and the positions of the occluder and adjacent structures were evaluated with Doppler transthoracic echocardiography (TTE). Pulmonary arteriography was performed before and after occlusion of the ASD (excluding the potential obstruction of RPV in the levophase). After the procedure, all patients received prophylactic antibiotics for 3 days and $200 \mathrm{mg} /$ day of aspirin for 6 months. All patients returned home after 3 days of observation in the heart ward.

\section{Follow-up}

For each patient, a chest radiograph, an electrocardiogram, and a TTE were recorded at 1 day, 1 month, and serially at 3- to 6-month intervals.

\section{Statistical Analysis}

The data in the study are expressed as mean \pm standard deviation for continuous variables and as number and percentage for categoric variables. The results before and after occlusion were compared using paired-sample $t$ tests. Statistical software used in this study was SPSS 16.0 (SPSS Inc Chicago, Ill).

\section{RESULTS}

\section{Clinical Characteristics}

Transcatheter closure was performed successfully in all patients, and Table 1 shows their clinical characteristics. Of 7 patients, 6 had a single defect, and 1 had 2 defects (the posterior ASD was $13 \mathrm{~mm}$ without RPV rim, and another ASD was $9 \mathrm{~mm}$ with sufficient rims). The size of the ASD (no RPV rim) was $13.4 \pm 2.2 \mathrm{~mm}$ (measured with TTE), the balloon-stretched diameter of ASD was $20.2 \pm 3.2 \mathrm{~mm}$ in the personalized heart model, and the 

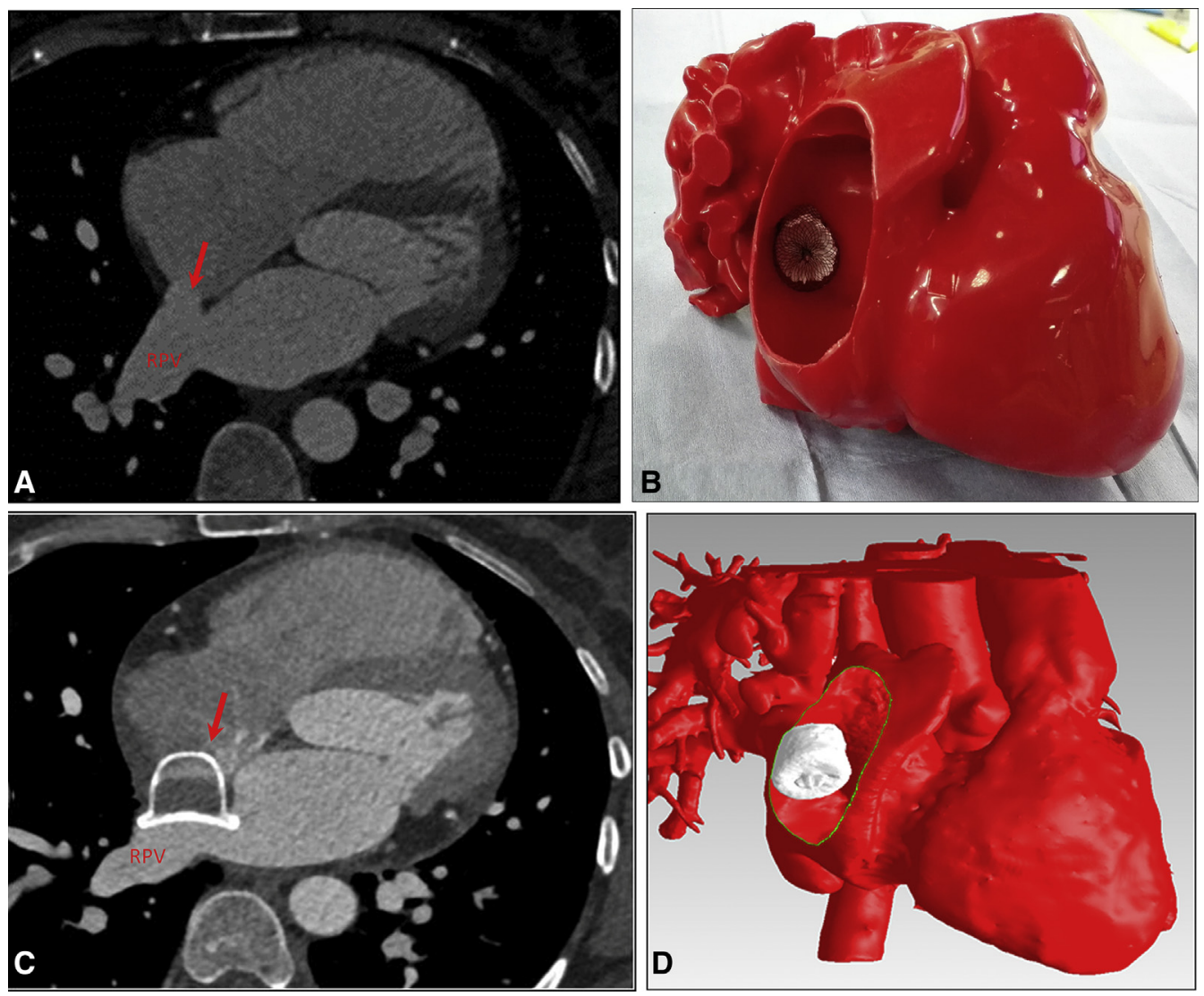

FIGURE 1. A 45-year-old woman with secundum ASD and no rim to RPV. MSCT showed an ASD with no RPV rim (A). The size of the ASD was $15 \mathrm{~mm}$ (arrow), and the balloon-stretched diameter was $21.8 \mathrm{~mm}$ in the personalized heart model. The in vitro trial closure was performed successfully with a $26 / 28$ Cera (Lifetech Scientific Co, Ltd, Shenzhen, China) duct occluder (B). After successful transcatheter closure, repeat MSCT confirmed the complete occlusion of the ASD (arrow) without obstruction of RPV (C). In addition, mild pericardial effusion was detected. The position and morphology of the device were almost identical between preoperative trial occlusion and postocclusion (D, reconstructed 3D image in the view of right atrium). $R P V$, Right pulmonary vein.

size of the selected occluder was $24.3 \pm 3.1 \mathrm{~mm}$. Right heart catheterization showed that the ratio of pulmonary to systemic blood flow was $2.03 \pm 0.38$, and mild pulmonary arterial hypertension occurred in 4 patients. The mean pulmonary arterial pressure was $25.1 \pm 4.3 \mathrm{~mm} \mathrm{Hg}$. In all patients, in vitro closure was achieved with the duct occluder, and subsequent transcatheter closure was performed successfully with the occluder of identical size.
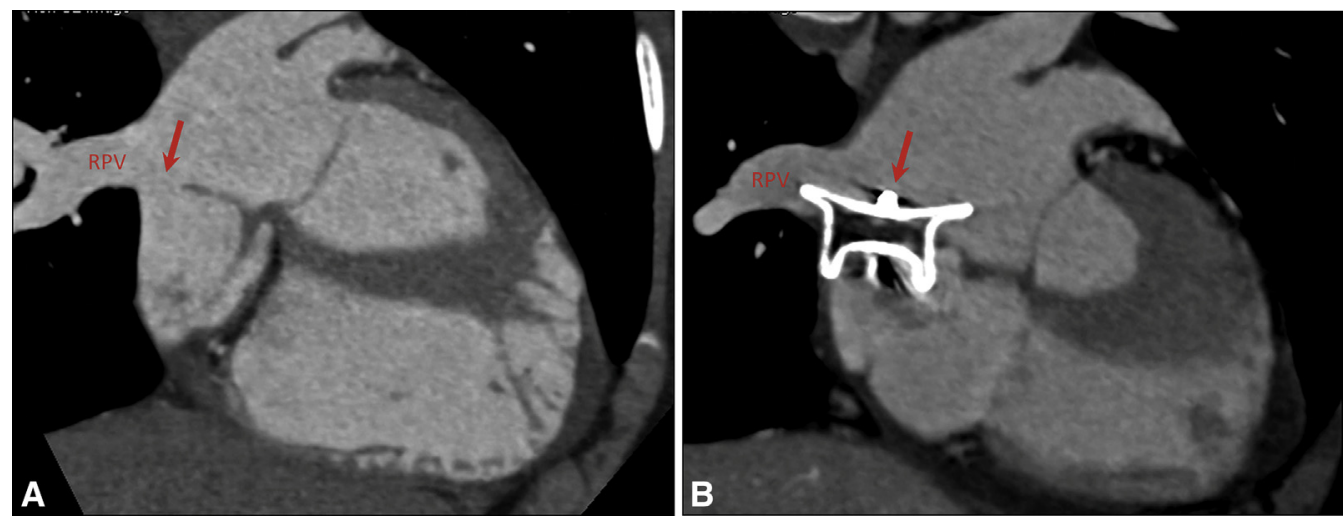

FIGURE 2. An 8-year-old boy with secundum ASD and no rim to the RPV. Preoperative MSCT indicated that there was no rim to the RPV, and the size was $9.8 \mathrm{~mm}$ (arrow, A). Successful transcatheter closure was performed with a 20/22 Cera duct occluder. The postoperative MSCT confirmed the good position of the device (arrow), and no obstruction occurred in the orifice of RPV (B). RPV, Right pulmonary vein. 
After transcatheter closure, the size was $21.4 \pm 3.5 \mathrm{~mm}$ in the waist of occluder (the narrowest segment of device). In the patients with 2 ASDs, another defect was closed with an atrial septal occluder (Video 3). The fluoroscopy time was $22.3 \pm 6.3$ minutes.

\section{Follow-up After Transcatheter Closure}

In 2 patients, mild pericardial effusion was identified after transcatheter closure ( 2 days and 7 days after closure, respectively) (Figure 1, $A-D$ ) and disappeared in 1 month and 3 months, respectively. In other patients, no complications occurred (Figure 2, $A$ and $B$ ). During the follow-up $(1.57 \pm 0.84$ years), the signs and symptoms had improved in all patients. TTE showed that there was no residual shunt at the atrial level, and no gradient occurred in the orifice of the RPV. In addition, no new arrhythmias were detected in the follow-up of the electrocardiogram. There was a significant decrease in the cardiothoracic ratio after transcatheter closure $(46.4 \% \pm 4.7 \%$ vs $53.1 \% \pm$ $5.0 \%, P=.012)$ and right ventricular end-diastolic dimension $(24.0 \pm 2.8 \mathrm{~mm}$ vs $31.7 \pm 4.3 \mathrm{~mm}$, $P=.002)$. Meanwhile, the increase was identified in the left ventricular end-diastolic dimension $(45.3 \pm 4.2 \mathrm{~mm}$ vs $40.0 \pm 5.4 \mathrm{~mm}, P=.001$ ).

\section{DISCUSSION}

In ASDs with no RPV rim, our findings suggested that transcatheter closure was feasible and safe using the duct occluder. With the aid of the personalized heart model, in vitro trial occlusion contributed to guide the selection of appropriate occluders and to evaluate the device stability preoperatively. Although the study involved the off-label use of a duct occluder, the preliminary result was satisfactory. To our knowledge, this is the first report to investigate the off-label use of a duct occluder in the transcatheter closure of an ASD with no RPV rim.

It is usually accepted that the current atrial septal occluder is not suitable for ASDs with no RPV rim, for which the double-disc occluder is unstable. ${ }^{1-7}$ In this research, surgical repair was suggested for these patients; however, they opposed surgical correction intensely and insisted on attempted transcatheter closure. Therefore, we investigated the feasibility of transcatheter closure in ASDs with no RPV rim. To avoid the unnecessary risks and identify the appropriate device, in vitro measurement and trial occlusion were performed preoperatively in a personalized heart model, as we described previously. ${ }^{8}$ In the elastic rubber model, different available devices were tested to determine the most suitable device, and the off-label use of the duct occluder proved feasible in these special ASDs. Subsequent transcatheter closure was also performed successfully in all patients, and the short-tomedium results were satisfactory.
In this research, occluder selection was based on the personalized heart model, and the size of the ASD was determined with the in vitro balloon-stretched diameter. The selected occluder was $4 \mathrm{~mm}$ larger than the in vitro balloon-stretched diameter in the study. Because of the lack of related research, there was no reference standard in the selection of device for patients with ASDs and no RPV rim, and it was mainly based on personal experience in the research. Mild pericardial effusion was identified in 2 patients and disappeared subsequently with medical therapy (within 3 months). We speculated that it was probably associated with the close contact between the duct occluder and the atrial wall/RPV. In other patients, the ASD was closed completely with no complications. The potential obstruction of the RPV was also excluded with pulmonary arteriography and TTE. Although the outcome was good, further studies are needed to evaluate this method beyond the indication and determine the most appropriate device size for these patients.

\section{Study Limitations}

Only a small number of patients were included in the research. To generalize the results of this study, a large population of patients are essential. Limited by the available device size (the maximal size was only $32 \mathrm{~mm}$ ), only the small to medium ASDs were included in this research. The results of this research are preliminary, and the long-term impact of this technique on the patency of the $\mathrm{RPV}$ is still unclear.

\section{CONCLUSIONS}

In ASDs with no RPV rim, transcatheter closure is feasible and safe with the off-label use of a duct occluder. By using the personalized heart model, in vitro measurement and trial occlusion provided an excellent method for preoperative evaluation. Further research is needed to investigate the long-term outcome.

\section{Conflict of Interest Statement}

Authors have nothing to disclose with regard to commercial support.

The authors thank Lu Xiangfeng for help with the statistical analysis, and Gao Yimin, Zhang Li, and Li Muzi for help with the TTE examination.

\section{References}

1. Du ZD, Hijazi ZM, Kleinman CS, Silverman NH, Larntz K, Amplatzer Investigators. Comparison between transcatheter and surgical closure of secundum atrial septal defect in children and adults: results of a multicenter nonrandomized trial. J Am Coll Cardiol. 2002;39:1836-44.

2. Butera G, De Rosa G, Chessa M, Rosti L, Negura DG, Luciane P, et al. Transcath eter closure of atrial septal defect in young children: results and follow-up. J Am Coll Cardiol. 2003:42:241-5.

3. Geva T, Martins JD, Wald RM. Atrial septal defects. Lancet. 2014;383:1921-32. 
4. Valente A, Rhodes JF. Current indications and contraindications for transcatheter atrial septal defect and patent foramen ovale device closure. Am Heart J. 2007; 153:81-4.

5. Du ZD, Koenig P, Cao QL, Waight D, Heitschmidt M, Hijazi ZM. Comparison of transcatheter closure of secundum atrial septal defect using the Amplatzer septal occluder associated with deficient versus sufficient rims. Am J Cardiol. 2002;90:865-9.

6. Kijima Y, Akagi T, Takaya Y, Taniguchi M, Nakagawa K, Kusano K, et al. Deficient surrounding rims in patients undergoing transcatheter atrial septal defect closure. J Am Soc Echocardiogr. 2016;29:768-76. - Kim MS, Hansgen AR, Wink O, Quaife RA, Carroll JD. Rapid prototyping: a new tool in understanding and treating structural heart disease. Circulation. 2008;117:2388-94.

8. Yan Chaowu, Li Hua, Sun Xin. Three-dimensional printing as an aid in transcatheter closure of secundum atrial septal defect with rim deficiency: in vitro trial occlusion based on a personalized heart model. Circulation. 2016; 133:e608-10.

9. Yamasaki Y, Nagao M, Kawanami S, Kamitani T, Sagiyama K, Yamanouchi T, et al. One-stop shop assessment for atrial septal defect closure using 256-slice coronary CT angiography. Eur Radiol. 2017;27:697-704.

Key Words: duct occluder, secundum atrial septal defect, 3-dimensional printing, transcatheter closure 